\title{
Atypical dopa responsive parkinsonism in a patient with megalencephaly, midbrain Lewy body disease, and some pathological features of Hallervorden-Spatz disease
}

\author{
P J Tuite, J P Provias, A E Lang
}

\begin{abstract}
A 38 year old patient with megalencephaly, mental retardation, and lifelong tremor developed levodopa responsive parkinsonism in his mid-30s followed by the appearance of dyskinesiae, motor fluctuations, hallucinations, and dementia. Brain MRI showed, as well as other changes, iron deposition in the globus pallidus, substantia nigra, and the pulvinar of the thalamus. Postmortem examination disclosed depigmentation of the substantia nigra pars compacta with neuronal loss, gliosis, and Lewy body formation. Axonal dystrophic spheroids, neuronal loss, calcification, and iron deposition were found in the substantia nigra pars reticulata. Less severe changes without neuronal loss were seen in the globus pallidus. This combination of megalencephaly with neuroaxonal changes predominently in the pars reticulata and Lewy body degeneration isolated to the substantia nigra pars compacta has not been previously reported.
\end{abstract}

(F Neurol Neurosurg Psychiatry 1996;61:523-527)

Keywords: Hallervorden-Spatz disease; Lewy body disease; parkinsonism; dystrophic axons

Hallervorden-Spatz disease and Parkinson's disease are two of the many causes of parkinsonism, each with a distinct neuropathology. Ten per cent of patients with HallervordenSpatz disease are said to have pathological features of both-that is, axonal spheroids and Lewy bodies. ${ }^{1}$ We report a patient with megalencephaly and dopa responsive parkinsonism who had pathological features, primarily involving the substantia nigra, reminiscent of both Hallervorden-Spatz disease and Parkinson's disease. The concurrence of these features (axonal spheroids, pigmentation, Lewy bodies) is not unique; however, the distribution and severity of changes are distinct. This specific constellation of findings and their association with megalencephaly have not been previously reported.

\section{Case report}

A 38 year old left handed man with congenital macrocephaly and mild mental retardation was referred to us at 35 years of age because of progressive and disabling tremor which had been present since childhood. The tremor predominantly involved his left hand and leg and impaired his ability to write and perform other activities of daily living. He denied slowness in performing activities and other motor impairments. There had been no exposure to neuroleptic medications and no history of encephalitis. His family history was significant in that his mother was diagnosed with "normal pressure hydrocephalus". In her 60 s she developed parkinsonism and a few years before her death at the age of 70 she became demented. No necropsy was performed.

His initial examination disclosed a masked facies, slight hypophonia, and normal eye movements. No Kayser-Fleischer rings were seen. A resting tremor with persistence with posture was prominent in the left arm and was present to a lesser degree in the left leg. A fine action tremor was present in the right arm. Moderate cogwheel rigidity and bradykinesia were found on his left side with mild involvement on the right. There was no dysmetria or ataxia. On ambulation there was decreased arm swing and he had normal postural stability.

Brain MRI T2 weighted images disclosed hypointensities, consistent with iron deposition, in the globus pallidus, substantia nigra, and the pulvinar of the thalamus. T2 weighted hyperintensities were seen lateral to the occipital horns of the lateral ventricles, and to a lesser extent there was involvement of the corticospinal pathways in the internal capsule and in the midbrain cerebral peduncles. No hydrocephalus was noted. $6-\left[{ }^{18} \mathrm{~F}\right]$ fluoro-L-dopa (Fdopa) PET showed normal caudate uptake and reduced bilateral putamenal uptake more pronounced on the right than the left.

Various medical and surgical treatments were employed in the hope of alleviating his disabling tremor. Propanolol, titrated to 240 mg per day, failed to change the tremor and it "worsened" on $300 / 75 \mathrm{mg}$ of levodopa/ carbidopa per day. Trials of primidone and 
Figure 1 MRI of brain: axial section $T 2$ weighted images at the level of the midbrain/diencephalic junction $(A)$ and the level of the midbrain (B). Note the prominent

hypointensity of the

substantia nigra, which is much darker than the red nucleus $(A)$. Bilateral

hyperintensities are present in the region of the corticospinal tract pathways in the cerebral peduncles. (C) MRI of brain: axial section $T 2$ weighted image at the level of the midthalamus. The globus pallidus and the pulvinar of the thalamus are hypointense.

Hyperintensities are seen in the internal capsules bilaterally, and lateral to the occipital horns of the lateral ventricular system. Postoperative changes from two prior thalamotomies are visible hypointense lesion) in the right thalamus.

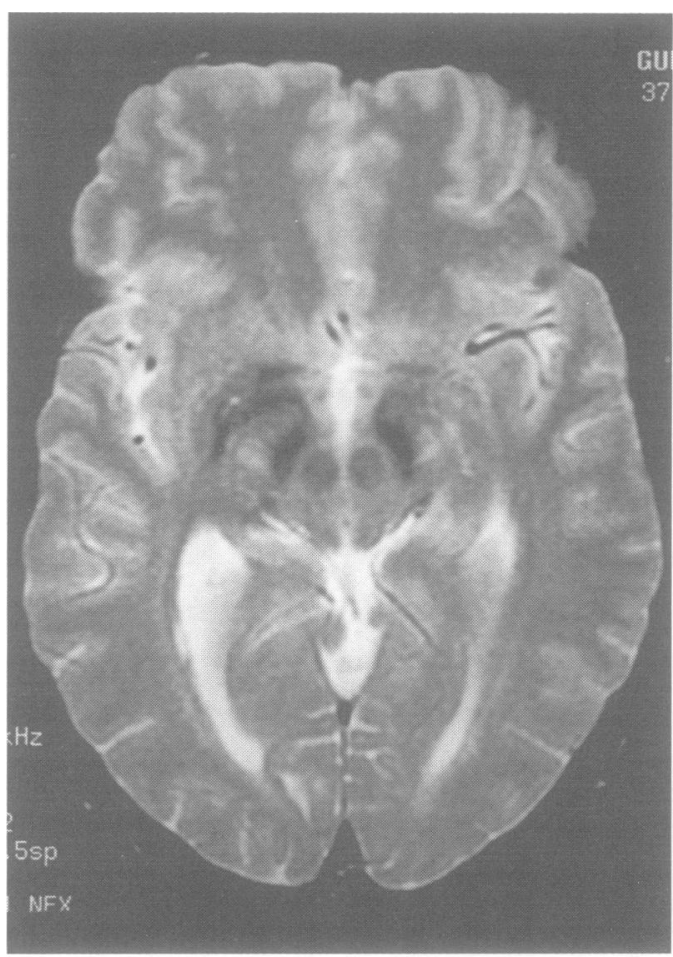

A

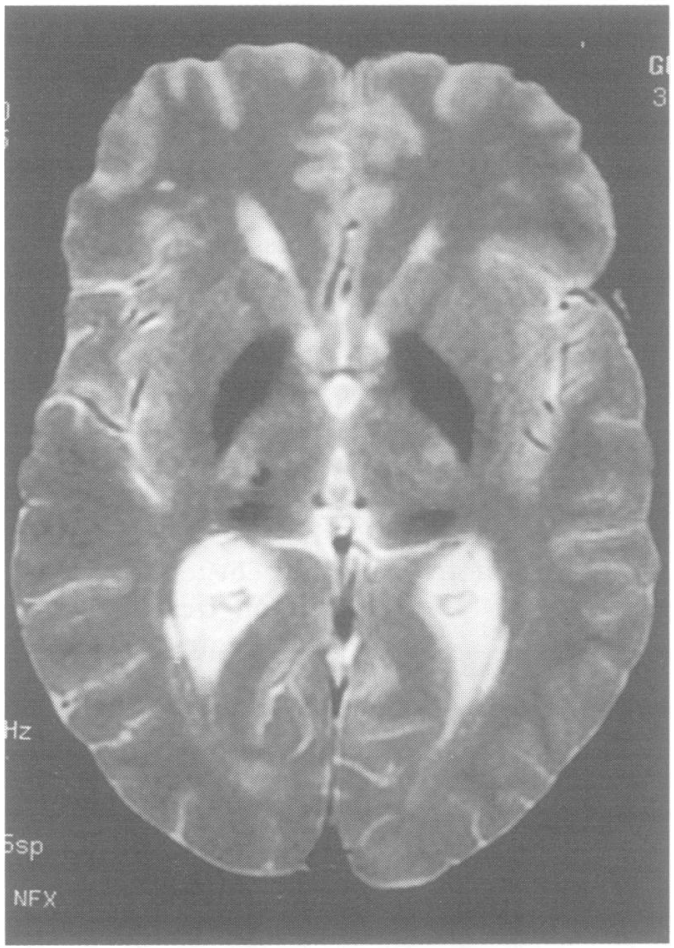

trihexyphenidyl also failed to diminish the tremor. He subsequently underwent a right thalamotomy which completely resolved his tremor for six days but then it returned to its preoperative severity. Four months later, a second right thalamotomy did not change the tremor or his rigidity but resulted in left hemiparesis and hemineglect.

Brain MRI performed 20 months after the initial study showed changes similar to the first MRI as well as the right thalamotomy lesion (fig $1 \mathrm{~A}-\mathrm{C}$ ).

A second trial with levodopa/benserazide greatly improved his motor function. When

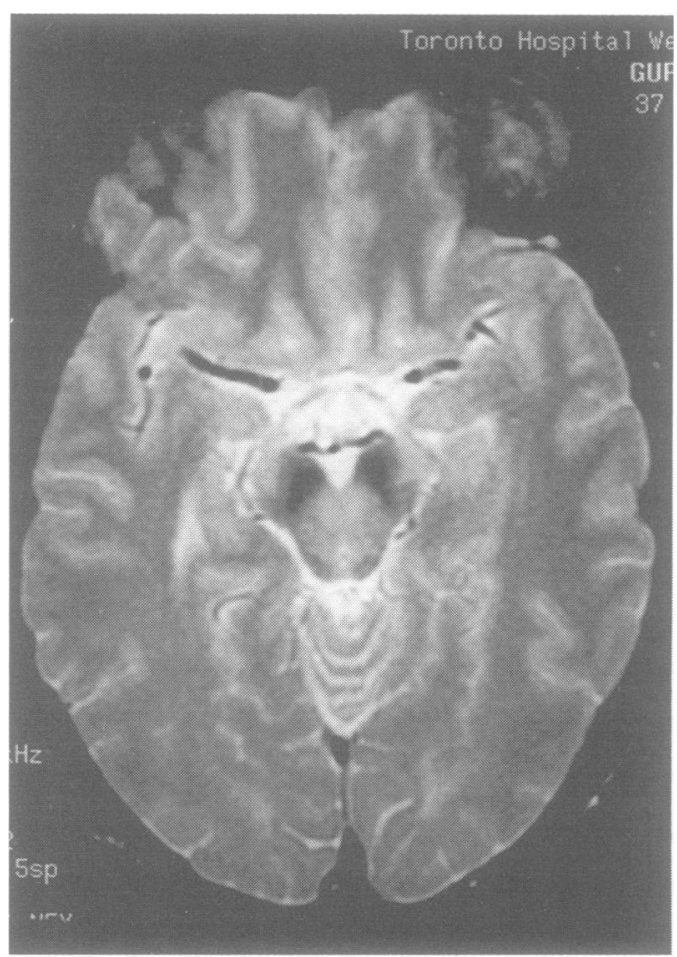

$B$

levodopa was increased to $1600 \mathrm{mg}$ per day and $50 \mathrm{mg}$ of trihexyphenidyl was added the resting tremor was eliminated for variable periods. With time he developed typical levodopa induced dyskinesiae of the face, trunk, and limbs with greater involvement on the right side of his body. He also developed a fluctuating response to medication and was immobile with a prominent tremor when he was in the "off" state. Severe postural instability eventually resulted in his becoming confined to a wheelchair. This was accompanied by cognitive changes including altered memory and concentration; his mini mental state examination score was 19/30. He developed frequent visual hallucinations and because of severe "off" periods he required continued use of high doses of medication (1300 mg levodopa and $50 \mathrm{mg}$ of trihexyphenidyl per day). As a result he was admitted to hospital and clozapine was titrated to $37.5 \mathrm{mg}$ per day, but he still had persistent hallucinations. $\mathrm{He}$ also developed fevers without a change in his white cell count and negative blood cultures. Empirically, antibiotics were started for a presumptive urinary tract infection. On the day of his planned discharge he was found in bed asystolic and failed to respond to resuscitation.

\section{Pathology}

A complete general necropsy disclosed extensive pulmonary aspiration of vegetative material.

Neuropathological examination was performed on the brain, two peripheral nerves, and muscle specimens using routine stains, immunohistochemistry (neurofilament, $\beta-4$ amyloid, synaptophysin, and ubiquitin), and special histological stains (Congo red, Bielschowsky silver, Perl's, and Von Kossa). The table summarises the pathological findings. 


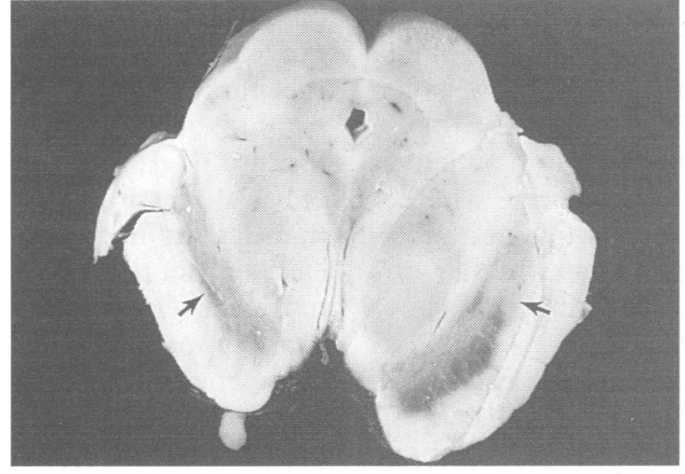

$A$

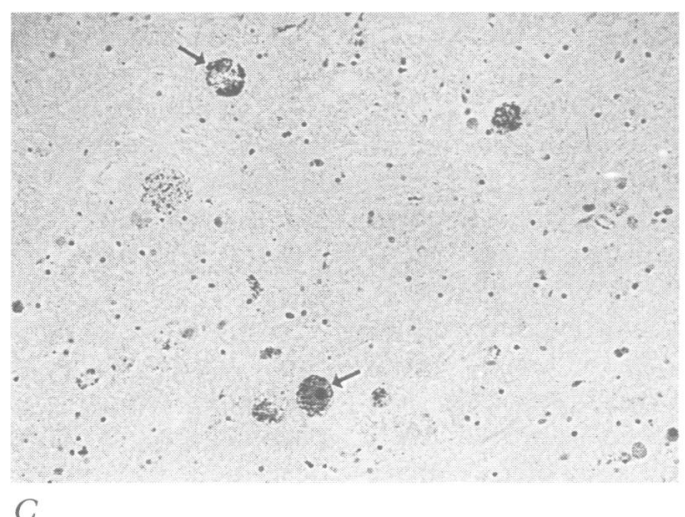

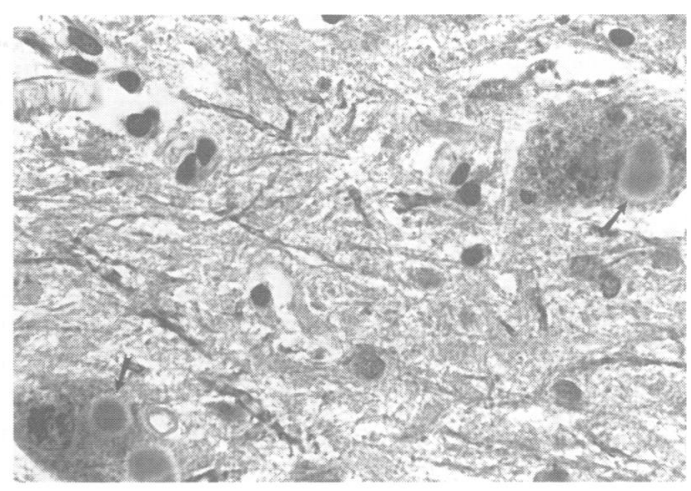

$B$

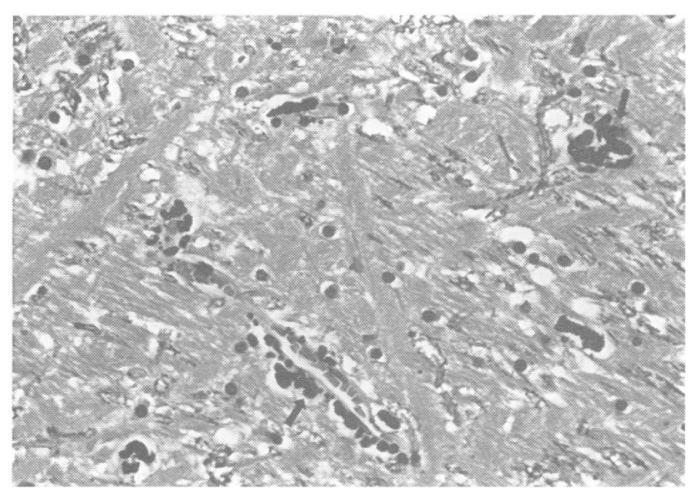

D

Figure 2. (A) Formalin fixed brain, transverse section through the midbrain at the level of the red nuclei. Note prominent brown discoloration of the substantia nigra, predominantly in the pars reticulata and more pronounced on the left side. There is also severe depigmentation of the pars compacta due to loss of neuromelanin containing neurons, most notably in the lateral aspects (arrows). (B) Histology of the substantia nigra. Remaining large neuromelanin containing neurons show classic cytoplasmic targetoid Lewy bodies (arrows). Haematoxylin and eosin originally $\times 400$. (C) Large granular axonal swellings (dystrophic axonal spheroids), shown here in the globus pallidus (arrows), were also seen in the substantia nigra pars reticulata (ubiquitin immunohistochemistry $(A B C)$ ). Originally $\times 250$ and 400 . (D) Areas of punctate mineralisation, often perivascular, were seen in the globus pallidus (arrows). Perl's positive staining indicates ferrous iron. Haematoxylin and eosin originally $\times 250$.

The brain weight was increased at $2180 \mathrm{~g}$. The brain was enlarged diffusely in a symmetric manner indicative of megalencephaly, and was without associated polymicrogyria. Coronal sections of the left hemisphere showed an incidental grey matter heterotopia in the white matter and a left occipital capillary telangiectasia. There was subtle histological evidence of cortical microdysplasia without other cortical pathology.

The striatum and brainstem structures, other than the substantia nigra, did not show any gross discoloration. The substantia nigra pars compacta, particularly the lateral aspects (fig 2A), showed asymmetric (right $>$ left) depigmentation due to moderate depletion of large neurons containing neuromelanin. This was accompanied by gliosis and prominent

Severity of pathological findings

\begin{tabular}{|c|c|c|c|c|c|c|c|}
\hline & $L B s$ & Gliosis & $\begin{array}{l}\text { Neuronal } \\
\text { loss }\end{array}$ & $D A s$ & NFTs & Other & $\mathrm{Fe} / \mathrm{Ca}$ \\
\hline SNpc & +++ & ++ & +++ & - & - & - & - \\
\hline SNpr & - & + & + & +++ & - & - & +++ \\
\hline$G P$ & - & - & - & Rare & - & - & ++ \\
\hline Putamen & - & - & - & Rare & Rare & - & - \\
\hline Thalamus & - & - & - & - & Rare & - & - \\
\hline Cortex & - & - & - & - & - & $\begin{array}{l}\text { Heteropia, } \\
\text { micro- } \\
\text { dysplasia }\end{array}$ & - \\
\hline
\end{tabular}

SNpc = Substantia nigra pars compacta; $\mathrm{SNpr}=$ substantia nigra pars reticulata; $\mathrm{GP}=$ globus pallidus; LBs = Lewy bodies; DAs = dystrophic axons; NFTs = neurofibrillary tangles; $\mathrm{Fe}=$ iron; $\mathrm{Ca}=$ calcium; $+++=$ severe; $++=$ moderate; $+=$ mild; $-=$ absent.
Lewy bodies in remaining neurons (fig 2B). The locus ceruleus, nucleus basalis of Meynert, and dorsal motor nucleus of the vagus were normal. Axonal swellings (dystrophic spheroid formation) were present primarily in the substantia nigra pars reticulata (fig 2C) and were accompanied by scattered microscopic brown pigmentation due to iron deposition and calcification. The pallidum was involved to a lesser degree and some of the deposits were present in a perivascular location (fig 2D). The other brainstem structures (pons, medulla), cerebellum, spinal cord, muscles (psoas and gastrocnemius), and peripheral nerve specimens (sciatic and sural) were normal.

\section{Discussion}

This patient's clinical course was suggestive of primary nigral neuronal loss because of his dopa responsive parkinsonism and subsequent development of motor fluctuations and levodopa induced dyskinesiae. ${ }^{2}$ However, the MRI showed prominent hypointensity of the pallidum and the substantia nigra on $\mathrm{T} 2$ weighted images, which suggested a diagnosis other than idiopathic Parkinson's disease. ${ }^{3}$

The pathological findings reaffirmed that this was not typical Parkinson's disease. To our knowledge, no case of megalencephaly has been reported with either dystrophic axons or 
Lewy bodies. Rather, patients with either or both of these features usually have small, atrophic brains. Thus the association between the megalencephaly (idiopathic?) and these "neurodegenerative" changes in our patient is unique. It is impossible to determine whether there was an aetiological or pathogenetic relation between these features or whether the combination was a mere coincidence.

Megalencephaly is defined as a brain that weighs above the 98th percentile and arises due to "metabolic" or "anatomical" causes. ${ }^{4}$ This patient had no evidence of a metabolic cause such as a gangliosidosis, a mucopolysaccharidosis, or a sulphatidosis. Anatomical megalencephaly is attributed to an increase in the number or the size of cells and can be associated with neurological disorders such as Alexander's and Cannavan's disease. Inherited (autosomal dominant or recessive) and sporadic (idiopathic) megalencephaly are also included in the anatomical causes and these are usually not associated with other neurological syndromes. As best it could be determined that there was no one else in the family with a large head. The focal cortical dysplasia and focal heterotopia have been reported with megalencephaly. ${ }^{5}$ However, they often coexist with evidence of severe neuronal migrational abnormalities - that is, pachygyria or polymicrogyria-which were not present in our patient. The cortical dysplasia does not account for the megalencephaly itself, but may represent the basis of this patient's mental retardation.

Lewy bodies are eosinophilic cytoplasmic inclusions composed partially of cytoskeletal protein with a high neurofilament content and polyubiquitin chains. ${ }^{6}$ Dystrophic axons, which are also ubiquitin-reactive, are composed of accumulated cytosolic and cytoskeletal components that result in the swelling of distal or terminal axons ("spheroids") probably due to defective axonal transport. ${ }^{7}$ The presence of Lewy bodies in the substantia nigra accompanied by gliosis and neuronal loss is consistent with a diagnosis of idiopathic Parkinson's disease although this inclusion may occur in other cerebral disorders. ${ }^{1}$ The nigral pathology suggestive of Parkinson's disease also correlates with many of the patient's clinical features. However, Lewy bodies were not found in structures that are often affected in Parkinson's disease (for example, hypothalamus, septal nuclei, locus ceruleus, dorsal motor nucleus of the vagus), or in diffuse Lewy body disease.

Dystrophic axons have been reported in Parkinson's disease but not to the extent or severity found in our case. ${ }^{1}$ More abundant dystrophic axons are typically associated with neuroaxonal dystrophies (Seitelberger's disease) and Hallervorden-Spatz disease, ${ }^{7}$ although in these disorders, dystrophic axons are usually present diffusely. In HallervordenSpatz disease, dystrophic axons are typically found in the spinal cord, the dorsal medullary region, the cuneate and gracile nuclei, the area postrema, the cerebral cortex, the pars reticulata, and the most severe changes are in the pallidum, particularly the internal segment. ${ }^{7}$ In our case dystrophic axons primarily involved the pars reticulata with a few scattered dystrophic axons found in the pallidum. The focality of dystrophic axons suggests a different sequence or pathophysiological process than occurs in typical Hallervorden-Spatz disease or neuroaxonal dystrophy, possibly one more closely linked to the pathology in the pars compacta.

In Hallervorden-Spatz disease, the coexistent pathology of the reticulata and the globus pallidus interna is not unexpected as both structures are embryologically related and any genetic or metabolic defect would presumably exist in their primordial cells. However, Kessler $e t a^{\mathcal{B}}$ have reported a case with selective pallidal involvement, which they include in the more general category of "HallervordenSpatz syndrome". This focal pallidal pathology is, in a sense, enantiomeric to that of our patient with more "selective" reticulata findings.

Although the nature of these combined features in our case is unique, the concurrence of the more typical Hallervorden-Spatz disease pathology and Lewy bodies have been described. ${ }^{9}$ In these patients, Lewy bodies have either been limited to the pars compacta or have been more widespread as in diffuse Lewy body disease. In some cases Lewy bodies were accompanied by neuronal loss and gliosis in the pars compacta, features which are not a part of typical Hallervorden-Spatz disease. However, all had accompanying HallervordenSpatz disease pathology (both dystrophic axons and mineralisation) involving both the pars reticulata and pallidum, the pallidum typically being most severely affected. None have had pathological changes similar to our patient. Many have had parkinsonism, some presenting during childhood or early adulthood. ${ }^{10-22}$ Some were treated with levodopa and responded ${ }^{14161819}$ whereas others failed to benefit. ${ }^{192122}$ Theoretically, the extent of the pallidal changes may have differed in these subgroups at the time of levodopa trial, thereby explaining the different levodopa responsiveness. We think that appreciable degeneration of the pars compacta combined with the lack of pallidal neuronal loss and gliosis in our patient explains why there was a response to levodopa.

In summary, our patient presents the unique combination of nigral degeneration with Lewy bodies and dystrophic axons, predominantly involving the substantia nigra, and megalencephaly. Further careful observation and research will be necessary to determine why this combination of cytoskeletal derangements occurs and its relation to the more typical forms of Parkinson's disease and Hallervorden-Spatz disease. The presence of megalencephaly is also of considerable interest, but is of uncertain relevance.

This work was partially supported by the National Parkinson's Foundation, Miami, Florida. We thank Shelley Malton for her assistance in preparing the manuscript and Dr E S Garrett for performing the F-dopa PET. 
1 Lewy bodies. In: Lowe J, Calne DB, eds. Neurodegenerative diseases. Philadelphia: WB Saunders, 1994:51-69.

2 Nutt JG. Dyskinesia induced by levodopa and dopamine agonists in patients with Parkinson's disease. In: Lang $\mathrm{AE}$, Weiner WJ, eds. Drug induced movement disorders. Mount Kisco, New York: Futura Publishing Co, 1992: $281-314$

3 Rutledge JN, Hilal SK, Silver AJ, Defendini R, Fahn S. Study of movement disorders and brain iron by MR. A7NR Am ₹ Neuroradiol 1987;8:397-411.

4 DeMyer W. Megalencephaly: types, clinical syndromes and management. Pediatr Neurol 1986;2:321-8.

5 Gooskens RHJM, Willemse J, Biilsma JB, Hanlo PW. Megalencephaly: definition and classification. Brain Dev 1988;10:1-7.

6 Pollanen MS, Dickson DW, Bergeron C. Pathology and biology of the Lewy body. $\mathcal{F}$ Neuropathol Exp Neurol 1993;52:183-91.

7 Seitelberger F. Neuroaxonal dystrophy: its relation to aging and neurological diseases. Handbook of Clinical Neurology 1986;5:391-415.

8 Kessler $\mathrm{CH}$, Schwechheimer K, Reuther R, Born JA. Hallervorden-Spatz syndrome restricted to the pallidal nuclei. $\mathcal{F}$ Neurol 1984;231:112-6.

9 Lowe J, Mayer RJ. Annotation: ubiquitin, cell stress and diseases of the nervous system. Neuropathol Appl Neurobiol 1990;16:281-91.

10 Defendini R, Markesbery WR, Mastri AR, Duffy PE. Hallervorden-Spatz disease and infantile neuroaxonal dystrophy: ultrastructural observations, anatomical pathology and nosology. F Neurol Sci 1973;20:7-23.

11 Gilman S, Barrett RE. Hallervorden-Spatz disease and infantile neuroaxonal dystrophy: clinical characteristics and nosological considerations. f Neurol Sci 1973;19: 189-205.
12 Jellinger K. Neuroaxonal dystrophy: its natural history and related disorders. Prog Neuropathol 1973;2:129-80.

13 Valsamis MP, Buha LM, Wisniewski HM. Coexistent infantile neuroaxonal dystrophy and "senile changes" in a young adult: a clinical, light and ultrastructural microscopic study. F Neuropathol Exp Neurol 1973;32:167.

14 Dooling EC, Schoene WC, Richardson EP. HallervordenSpatz syndrome. Arch Neurol 1974;30:70-83.

15 Vuia O. Neuroaxonal dystrophy, a juvenile-adult form. Clin Neurol Neurosurg 1977;79:307-15.

16 Williamson K, Sima AA, Curry B, Ludwin SK. Neuroaxonal dystrophy in young adults: a clinicopathological study of two unrelated cases. Ann Neurol 1982;11: 335-43.

17 Kalyanasundaram S, Srinivas HV, Deshpande DH. An adult with Hallervorden-Spatz disease: clinical and
pathological study. Clin Neurol Neurosurg 1980;82:245-9.

18 Alberca R, Rafel E, Chinchon I, Vadillo J, Navarro A. Late onset Parkinsonian syndrome in Hallervorden-Spatz disonset Parkinsonian syndrome in Hallervorden-Spatz
ease. $\mathcal{f}$ Neurol Neurosurg Psychiatry 1987;50:1665-8.

19 Eidelberg D, Sotrel A, Joachim C, et al. Adult onset Hallervorden-Spatz disease with neurofibrillary pathology. Brain 1987;110:993-1013.

20 Gaytan-Garcia S, Kaufmann JCE, Young GB. Adult onset Hallervorden-Spatz syndrome or Seitelberger's disease with late onset: variants of the same entity? A clinicopathological study. Clin Neuropathol 1990;9:136-42.

21 Odawara T, Iseki E, Yagishita S, et al. An autopsied case of juvenile parkinsonism and dementia, with widespread occurrence of Lewy bodies and spheroids. Clin Neuropathol 1992;11:131-4.

22 Sugiyama H, Hainfellner JA, Schmid-Siegel B, Budka H. Neuroaxonal dystrophy combined with diffuse Lewy body disease in a young adult. Clin Neuropathol 1993;12: 147-52. 